\title{
DISCURSOS VISUALES EN LOS MUSEOS ARQUEOLÓGICOS PROVINCIALES DE ANDALUCÍA. PROPUESTA EXPOSITIVA DE LA TUMBA DEL GUERRERO EN EL MUSEO DE MÁLAGA
}

\author{
VISUAL DISCOURSES IN THE PROVINCIAL ARCHAEOLOGICAL \\ MUSEUMS OF ANDALUSIA. EXHIBITION PROPOSAL OF THE \\ WARRIOR'S TOMB IN THE MUSEUM OF MALAGA
}

\author{
Julia García González \\ (Universidad de Málaga, España) \\ juliagargon@uma.es
}

Recibido: 8 de octubre 2020 / Aceptado: 8 de febrero 2021

\begin{abstract}
Resumen: Partiendo de la premisa de que los bienes y sus contenedores son elementos vivos y fundamentales en la sociedad del s. XXI, en el presente artículo, pretendemos mostrar el panorama regional de los discursos expositivos y casuísticas de los museos arqueológicos provinciales para centrarnos en el Museo de Málaga y la presentación de un hallazgo que difiere y modifica el discurrir expositivo de dicho espacio. Así, los objetivos de este trabajo son: la contextualización de la presencia de secciones de arqueología en diferentes museos provinciales andaluces; el estudio de la musealización de la Tumba del Guerrero junto a su contextualización; y la plasmación de una propuesta expositiva tras las últimas publicaciones de los estudios sobre el objeto de investigación ya que aportan interesantes datos que pueden ser incluidos en el discurso expositivo.
\end{abstract}

Palabras clave: Tumba del Guerrero; musealización; Museo de Málaga; arqueología; Andalucía.

\begin{abstract}
Based on the premise that archaeological remains and their containers are living elements and a fundamental part of the society in the 21 st century, in this article we intend to present the regional landscape of exhibition discourses and casuistry of different archaeological provincial museums. We will focus on the Museum of Malaga and the showcase of a finding that differs and modifies the exhibition plan of said space. Thus, the objectives of this article are the contextualisation of the archeological sections present in different provincial museums in Andalusia; the study of the current musealisation of the Warrior's Tomb and parallel studies; and the presentation of the exhibition proposal that
\end{abstract}


suggests the addition of data extracted from the latest studies on the object to the exhibition discourse.

Keywords: Warrior's Tomb; musealization; Malaga Museum; archeology; Andalusia.

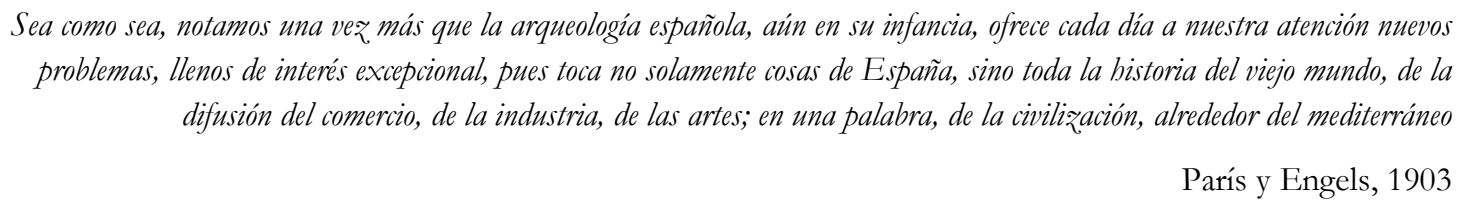

\section{Introducción}

Andalucía posee museos provinciales en su vertiente arqueológica con discursos expositivos y trayectorias muy diversas. A continuación, pretendemos presentar sucintamente el devenir de cada uno para detenernos en aquellos que presentan una sección de arqueología. A partir de este punto nos adentraremos en los elementos comunes y las diferencias entre ellos para, finalmente, prestar atención al discurrir del Museo de Málaga y su configuración expositiva.

Tras ello analizaremos la museografía de la Tumba del Guerrero. Se trata de un hallazgo producto de la realización de una excavación de urgencia acometida en la ciudad de Málaga en el año 2012. Esta constituye, con todos los elementos que la conforman, una pieza clave del discurso de la sección arqueológica del Museo de Málaga y, entre otras cuestiones, destaca porque su planteamiento museográfico difiere del resto de la colección al presentar en un mismo espacio su continente y su contenido.

Para finalizar planteamos una propuesta expositiva de la Tumba del Guerrero motivada por la presentación de los resultados derivados del estudio exhaustivo de todos los elementos constituyentes del bien.

\section{Los Museos Arqueológicos en Andalucía}

En 1857, la Ley General de Instrucción Pública, inicia un recorrido con la recomendación de la creación de un museo provincial de antigüedades en capitales de provincia, que nos permite, a día de hoy, la investigación, el disfrute, el conocimiento y la conservación de nuestro pasado. 
El siguiente hito, en pro de conseguir dichos objetivos, se produce en 1867 con la aprobación, el 20 de marzo de ese mismo año, de la creación del Museo Arqueológico Nacional. De esta manera se inicia también la oportunidad de apertura de una red de museos arqueológicos provinciales públicos y de titularidad estatal que constituye el germen de los actuales Museos Provinciales en Andalucía. Estos, teniendo como punto de partida la cultura material conservada gracias al trabajo desarrollado por las Comisiones Provinciales de Monumentos Histórico Artísticos (1844), han sufrido un discurrir disímil.

A partir de esa fecha, y siendo conscientes de la singularidad de cada uno de ellos, se comienzan a configurar museos arqueológicos provinciales que derivan de las secciones de antigüedades o Gabinetes de Antigüedades dependientes de los museos de Bellas Artes, las Comisiones provinciales de monumentos, o las diputaciones, y que en las provincias andaluzas poseen una presencia destacada fruto del devenir histórico de toda la comunidad en la que se han sucedido y convivido diversas culturas. Así, en 1868 se crea El Museo Arqueológico Provincial de Córdoba, en 1879 se produce la apertura del Museo Arqueológico Provincial de Granada y también del Museo Provincial de Antigüedades de Sevilla y, en 1933, el tan esperado Museo Arqueológico Provincial de Almería.

El Museo Arqueológico de Sevilla se crea en 1879. Ahora bien, no será hasta 1946 en que la colección se completa al integrarse las piezas del Museo Municipal de Sevilla en el arqueológico. Destaca por germinar dentro de los gabinetes humanistas o studioli del siglo XVI y desarrollarse hasta el punto de presentarse en la actualidad como un museo de referencia dentro de la disciplina arqueológica (Navarro et al., 2007).

Tanto el Museo Arqueológico Provincial de Córdoba como el Museo Arqueológico de Granada, junto al ya referido museo sevillano, son unos de los primeros fundados en España tras la apertura del Museo Arqueológico Nacional. Ambos poseían una dilatada trayectoria en cuanto a su colección pues parten de gabinetes de antigüedades, en el caso de Granada, de colecciones de antigüedades creadas por las comisiones de monumentos en la década de los 40 del s. XIX de manera autónoma, o bien, como en el caso de Córdoba, dependiendo del Museo de Bellas Artes. Del mismo modo, tanto el Museo Arqueológico de Córdoba como el Museo Arqueológico de Granada situaron su sede en palacetes renacentistas de primer orden: el Palacio de los Páez de Castillejo y la Casa de Castril respectivamente (Toro, 2017). Ahora bien, su historia reciente es distinta puesto que el Museo Arqueológico Provincial de Córdoba cuenta con un museo renovado y ampliado que ha sido capaz de incluir en su recorrido los restos arqueológicos del teatro romano que 
subyacen en su subsuelo, dotarlo de los servicios necesarios y presentarlo como un referente cultural de la ciudad. Frente a ello, el Museo Arqueológico Provincial de Granada, tras demasiados años cerrado, ha abierto sus puertas de manera parcial sin realizar la verdadera transformación tan necesaria y demandada por la sociedad.

El Museo Arqueológico Provincial de Almería es el último en configurarse debido, según Manuel Ramos (2017), a varias cuestiones. En primer lugar, a la inexistencia de un museo de bellas artes. En segundo lugar, a no aprovechar la vía seguida en la provincia de Málaga de apertura de un museo a partir de la academia artística local. En tercer lugar, a la dispersión de la colección Siret. Y, en cuarto lugar, a la inexistencia de un consolidado grupo intelectual preocupado por la tutela y difusión del patrimonio. Pese a todas estas trabas, afortunadamente, el museo abría sus puertas en 1933 aunque sin acoger la colección Siret para la que iba a ser creado. Además, ha entrado con fuerza en el s. XXI al convertirse, de un lado, en el primer museo arqueológico provincial andaluz en presentar una renovación integral y, de otro, en un referente museográfico, debido, entre otras muchas cuestiones, a la conexión que presenta su discurso expositivo entre pasado y manifestaciones o expresiones contemporáneas.

Al mismo tiempo surgen museos específicos de monumentos de primer orden como el Museo de Antigüedades de la Alhambra en 1873 que, en cierto modo, constituyen la base de los actuales Museos de Sitio de los diversos Conjuntos Arqueológicos de Andalucía y que posibilitan la permanencia de la cultura material próxima al contexto en el Museo del Conjunto Arqueológico de Madinat Al-Zahra en Córdoba o El Museo del Conjunto Arqueológico de Baelo Claudia en Cádiz. A los Museos Arqueológicos Provinciales y a los Museos Arqueológicos de Sitio asociados a la figura de Conjuntos arqueológicos, debemos añadir, los Museos Arqueológicos Municipales como el de Linares o Úbeda, ambos en la provincia de Jaén.

Ya hemos hecho referencia a los museos arqueológicos provinciales de Granada, Córdoba, Sevilla y Almería, sin embargo, en las provincias de Málaga, Cádiz, Huelva y Jaén, en la actualidad, observamos una realidad desemejante pues la cultura arqueológica se presenta dentro de la sección de arqueología. Ello significa que las piezas que acogen las secciones arqueológicas comparten institución con la sección de Bellas Artes conformando los museos provinciales de cada provincia. Ahora bien, como no todos han seguido el mismo recorrido, pues algunos, como por ejemplo el Museo de Málaga, se conforman a partir de la unión de dos museos independientes, es necesario que los analicemos brevemente a 
continuación, a excepción del Museo de Málaga en el que nos centraremos en el apartado tres por ser el que nos compete.

En el caso de Cádiz observamos la misma trayectoria que en el Museo de Málaga, es decir, la de dos museos en origen independientes: Museo Arqueológico y Museo de Bellas Artes, que terminan por fusionarse en 1970 formando el Museo de Cádiz e incorporando, a las secciones de Arqueología y Bellas Artes, la Sección de Etnología. Ahora bien, su origen es diverso pues, mientras el Museo de Bellas Artes inicia su andadura en 1852, no será hasta 1889 cuando el Museo Arqueológico se inaugure con motivo del descubrimiento del sarcófago fenicio antropomorfo de Punta de la Vaca, (De la Sierra, 2017).

En lo que respecta al Museo Provincial de Jaén cabe precisar que tiene su origen en la Diputación Provincial, concretamente dentro del Instituto de Estudios Giennenses que se funda en 1951 y se organiza por secciones: Arqueología y Bellas Artes. En 1963 la sección de arqueología se independiza conformando el Museo Arqueológico Provincial, pero, en 1969, se fusiona con el Museo de Bellas Artes, creado en la primera mitad del s. XX. De esta manera, a partir de la década de los 70 y hasta la actualidad, se concibe en su doble vertiente, (Chicharro, 2013) al igual que veremos que se produce con el Museo de Málaga.

En lo que respecta a Huelva habrá que esperar a 1920 en que se funde la institución. Esta surge desde sus inicios como Museo de Huelva y se estructura mediante tres secciones: Arqueología, Bellas Artes y Etnología, aunque «esta última no formará parte del Museo debido a la falta de espacio» (Guisande, 2017, p.316) pasando su contenido finalmente a la colección del Museo de Artes y Costumbre Populares de Sevilla. 


\section{El caso del Museo de Málaga y la musealización de la Tumba del Guerrero}

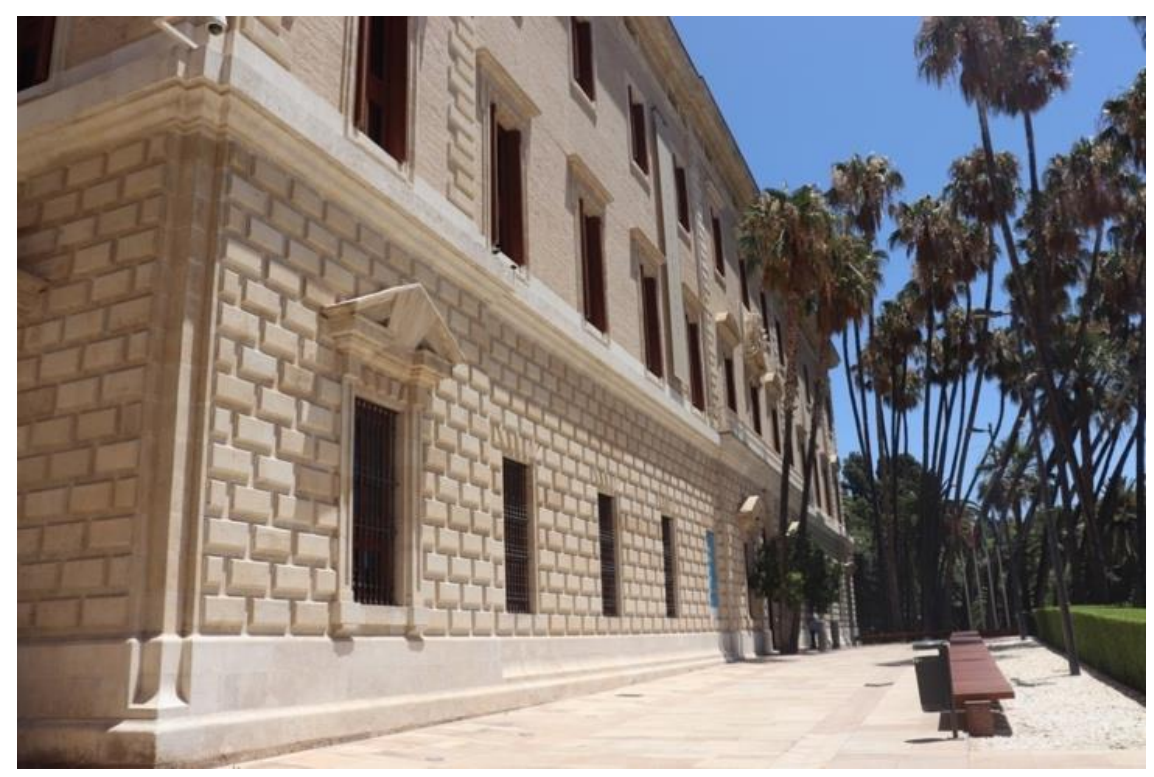

Fig. 1. Vista del Palacio de la Aduana, en la actualidad Museo de Málaga, elaboración propia, 2020.

El Museo de Málaga, en la actualidad de titularidad estatal y gestión autonómica, presenta un discurrir histórico tremendamente personal, pero, al mismo tiempo, con paralelismos con el resto de museos andaluces tanto en lo que respecta a su conformación como en lo que concierne a su historia mas reciente.

En 1945 se crea el Museo Arqueológico Provincial gracias a que los trabajos de la Alcazaba posibilitan su instalación en este espacio. Ahora bien, la colección no se constreñía estrictamente a la cultura material extraída de este enclave, sino que estaba también conformada por las piezas cedidas por los Marqueses de Casa Loring y que habían pertenecido al denominado Museo Loringiano sito en la Finca de la Concepción. A estas se agregaban las piezas de las colecciones privadas de Juan Temboury, Simeón Giménez y la Sociedad Malagueña de Ciencias.

En 1972 se produce la unión del Museo Provincial de Bellas Artes y el Museo Arqueológico Provincial, aunque estos seguirán en ubicaciones independientes. De este modo el Museo de Málaga, aunará mediante secciones, el Museo Arqueológico Provincial y el Museo Provincial de Bellas Artes abierto en 1916.

En 1984 se transfieren las competencias de gestión a la Junta de Andalucía dentro del nuevo marco que configuraba el Estatuto de Autonomía de Andalucía. La sección arqueológica cierra sus puertas en 1996 y, tan sólo un año mas tarde, lo hace su sección de 
Bellas Artes. A partir de ese momento la provincia queda vetada del disfrute de parte de su patrimonio. Siendo una problemática común a otros museos andaluces como el Museo Arqueológico de Granada que, desgraciadamente, ha tenido que lidiar con lo que podríamos determinar la «eterna clausura». Este hecho origina la creación de un movimiento ciudadano, dentro del cual se encuentra, junto a asociaciones, fundaciones, colegios profesionales o sindicatos, la plataforma «La Aduana para Málaga». La misma reivindica el derecho a acceder a su patrimonio, ya sea arqueológico o artístico, y motiva que, en el año 2005, los organismos competentes decidan la nueva apertura del museo en el Palacio de la Aduana [Fig.1] mediante cesión del edificio por parte del Ministerio de Cultura y que permite que se convierta en su sede. Tal y como escribe el profesor Santana se trata de:

« ...un inmueble administrativo construido entre los siglos XVIII y XIX que perdió su cubierta original, inclinada, en un pavoroso incendio en 1922. En el proyecto de rehabilitación como continente cultural se decidió devolverle su perfil original, aunque con una estética contemporánea, con el uso de grandes tejas metálicas [...], material poco apropiado para un inmueble destinado a custodiar obras de arte y construido en esta latitud, [...]. Las fachadas se rehabilitaron, se conservaron las bóvedas vaídas de la primera planta y de las escaleras principales. Es una intervención interesante donde destaca el lenguaje contemporáneo, si bien en algunos casos se han eliminado preceptos neoclásicos del inmueble original.» (Santana, 2019, p.547)

La intervención arquitectónica, no exenta de crítica (Gutiérrez de Pablo, 2013, p.252-256), corrió de manos de los arquitectos Fernando Pardo, Bernardo García Tapia y Ángel Pérez Mora. Por su parte, el equipo de Frade arquitectos, bajo la ejecución de Empty, se encargó del discurso museográfico, inserción del equipamiento y mobiliario.

El Museo, además de poseer múltiples servicios adaptados a las necesidades y demandas del público en la actualidad como, recepción, guardarropa, cafetería, restaurante o tienda, destaca por articularse mediante cuatro elementos que le otorgan personalidad. Nos referimos a: la disposición en la planta baja de un almacén visitable; la integración de los restos arqueológicos correspondientes a la muralla medieval de la ciudad dentro del museo a través de un pavimento acristalado dispuesto en la cafetería; y la diversificación de espacios, dedicando la primera planta a la sección de Bellas Artes y la segunda planta a la sección de Arqueología, ambas con un discurso museográfico propio.

En paralelo, en 2012, una intervención arqueológica de urgencia dirigida por David García González en los solares número 12- 38 de la calle Jinetes y números 15, 19, 23 y 27 de la calle Refino de Málaga, con una extensión en superficie de 3492,20m² (García González, 
2018, p.75), permite el conocimiento de la denominada, ya comúnmente, Tumba del Guerrero. Se trata de una tumba en cista protohistórica hallada junto a otras estructuras de períodos posteriores. Entre ellas podemos destacar un área de residencia almohade. En cuanto a los espacios de época moderna, para este estudio, consideramos necesario destacar la excavación, en la esquina suroeste del espacio en el que se interviene, de «una fosa con los restos óseos de tres mujeres» (García González, 2018, p.80-81) que, a partir de los resultados del análisis antropológico, ha permitido a los investigadores concluir que «... podemos encontrarnos ante las víctimas de una de las múltiples epidemias que asoló la ciudad de Málaga a lo largo de época Moderna». (García González, 2018, p.81) y que, por tanto, consideramos que aportan datos de primer orden en el conocimiento, gracias a la arqueología, de la historia reciente de la ciudad.

La tumba en cista esta realizada en piedra calcarenita, revestida de hiladas de mampostería de cantos, y se adscribe al s. VI a.C. En su interior y entorno se hallaron una serie de artefactos que han permitido conocer mejor a la persona enterrada y el rito de enterramiento. Así, en el nivel de colmatación de la tumba hallaron una serie de materiales cerámicos asociados con el ritual de enterramiento del individuo; en la cabecera de la tumba, el quemaperfumes de cerámica y el casco corintio; y en el interior, junto a los restos óseos, se documentaron restos de la posible cubierta de madera de la tumba, un anillo con escarabeo realizado en plata, oro y cornalina; un conjunto de armas (una moharra de hierro y un posible escudo); varillas de plata de un posible pergamino; una phiále de plata y restos de marfil (García González, 2018, p.75).

A continuación, pasamos a describir brevemente las partes de este conjunto pues, de este modo, pretendemos que el lector conozca la necesidad de mostrarlo en su plenitud.

En la cabecera de la tumba, concretamente «... sobre una hilada de cantos de piedra dispuestos sobre los sillares que conformaban la pared oeste de la estructura funeraria». (García González et al., 2018, p.270), se disponían el quemaperfumes y el casco corintio. El quemaperfumes cerámico de dos receptáculos contenía evidencias de carbón que esclarecen su uso dentro del ritual funerario asociado a la cultura fenicio-púnica. Además, a partir de su procesado informático, se han podido localizar restos del engobe rojo y un motivo de bandas concéntricas en la parte superior (García González et al., 2018, p.270-275).

A pocos centímetros del quemaperfumes se situaba una de las piezas mas aplaudidas de la tumba, y única en la Península Ibérica, nos referimos al casco corintio. Este fue inutilizado seguramente durante el ritual de enterramiento pues «las dos piezas cubre-mejillas 
aparecen "empujadas", forzadas hasta unirse entre sí cerrando todo el espacio para la boca, bajo el nasal» (García González et al., 2018, p.188) y constituye una importante arma defensiva pasiva y símbolo de ostentación. De hecho, presenta un programa decorativo complejo en el que destacan la palmeta de once pétalos en la parte frontal y las aves rapaces con las alas desplegadas pero asimétricas entre ellas a la altura de las sienes y en las carrilleras (García González et al., 2018, p.196).

Junto al casco, la tumba poseía otras armas como una moharra de lanza y un posible escudo circular de tipo aspis que configurarían «una panoplia de tipo hoplita, única hasta la fecha en la Península ibérica [...] con una datación en torno al segundo cuarto avanzado del s. VI a. C» (Quesada y García González, 2018, 146). En cuanto a la disposición en la tumba de las mismas, la moharra «se encontró sobre el suelo en la esquina suroeste de la cista, doblada casi en ángulo recto y parcialmente apoyada sobre la pared oeste [...] doblada (y por tanto inutilizada) intencionalmente como parte de un ritual» (Quesada y García González, 2018 , 146). En cuanto al escudo «antes del hallazgo de los restos óseos del inhumado [...] se documentó, en la mitad oeste, un gran número de fragmentos de lámina de bronce. Muchos de ellos presentaban decoración repujada» (Quesada y García González, 2018, 146). Además, tras la retirada de los restos óseos aparecieron varios objetos circulares que los investigadores plantean como parte de la misma pieza.

Otra pieza clave y única hasta el momento es el anillo con escarabeo de tipo egipcio hallado «a la izquierda de la cabeza del difunto» (Ortiz y Jiménez, 2018, 252) y que sería realizado «probablemente en Egipto, llevándose a cabo su entrada en la Península Ibérica posiblemente a través de un circuito fenicio» (Ortiz y Jiménez, 2018, 258) en los s. VII y VI a.C. Los materiales utilizados son oro para la montura, aleación con plata para el aro y escarabeo de cornalina. Este último fue tallado por ambas caras. En el anverso se muestra la anatomía del escarabajo y en el reverso una diosa sedente con cabeza de león que los investigadores asocian a Sejmet. Esta porta sobre su cabeza un disco solar con serpienteureo y sujeta el ojo-udjat con una mano mientras realiza un gesto de adoración con la otra. Asimismo, se observa un cartucho con escritura jeroglífica que no se ha podido identificar (Ortiz y Jiménez, 2018, 252).

A todo ello habría que añadir la presencia de dos varillas de plata rematadas en sus extremos por pomos hemisféricos que podrían utilizarse como «vástagos sobre los que enrollar un soporte de escritura» (Núñez, 2018, 232). 
Otro objeto de plata que conformaría el ajuar es la phíale situada, al igual que el anillo con escarabeo, a la izquierda de la cabeza del inhumado que, utilizada habitualmente como contenedor de líquidos, sería empleada durante el ritual de enterramiento «en el aseo del cadáver, como parte de los ritos previos al enterramiento, quizás para el lavado de las heridas y suciedad que pudiese cubrir el cuerpo» (García González y García Alfonso, 2018, 250). Concretamente presenta en el fondo la inserción de una pieza circulada soldada al mismo con un motivo decorativo que muestra una roseta central de ocho pétalos que rodean un botón central (García González y García Alfonso, 2018, 241-242)

Ahora bien, estos no serían los únicos objetos que conformarían el ajuar puesto que durante el trabajo de campo se documentaron a la derecha de la cabeza del individuo restos de marfil que formaría parte de un objeto que no se ha podido identificar. Pese a ello los investigadores, mediante un análisis de paralelos señalan que «La gran variedad de objetos elaborados en marfil incluye cajas, utensilios de cosmética, peines, cucharas o placas decoradas» (Lamarca et al., 2018, 266) y por tanto podría formar parte de alguno de estos objetos, aunque es imposible precisar cual.

Por último, nos detenemos en los restos óseos hallados. Estos mostraban desde el inicio un «delicado estado de conservación [...] y de lo complicado que podía ser su excavación y limpieza» (López, 2018, 302). Pese a ello se ha podido conocer que se trata de un enterramiento indirecto en el que el cuerpo se encontraba decúbito supino en posición primaria, aunque «... a causa de procesos tafonómicos de descomposición del cadáver y la paulatina colmatación de la tumba con sus propias características dinámicas, los restos óseos presentan ciertas disposiciones no anatómicas» (López, 2018, 304). Así, por ejemplo, el cráneo y la mandíbula se encontraron aplastados, fragmentados y rotados o las vértebras cervicales desplazadas, hechos que se producen por procesos tafonómicos relacionados con la descomposición del cadáver y el colapso de la cubierta sin detectarse «prácticas postsepulcrales tales como reapertura de la tumba, manipulación de los restos óseos, reducciones, ni reinhumaciones» (López, 2018, 307). El estudio antropológico ha concluido que se trata de «un individuo masculino, de algo más de 40 años, elevada estatura y fuerte desarrollo musculan (Jiménez y Laffranchi, 2018, 316) que «no sufrió carencias alimentarias durante su infancia» (Jiménez y Laffranchi, 2018, 316) y cuyas características óseas permitirían considerarlo un «guerrero».

La importancia a nivel internacional del hallazgo y la decisión de no mantener los restos in situ sino de tutelarlos y difundirlos en el Museo Provincial en proceso de renovación, 
motiva una reestructuración del Programa Expositivo del Museo de Málaga integrado en el Plan Museológico del mismo publicado en 2011 (Palomares, 2018, p.381). Este hecho repercute por tanto en la remodelación de la sección de Arqueología al decidirse que sea esta la ubicación definitiva de la tumba. Así, en la segunda planta, encontramos un discurso expositivo dividido en:

" ...ocho bloques expositivos, que se iniciaba en torno a las primeras colecciones arqueológicas de aficionados burgueses decimonónicos, específicamente aquellas reunidas por los marqueses de Casa-Loring en Málaga y de salamanca y Madrid [...] Le seguían dos bloques concomitantes destinados a la presentación del mas remoto pasado provincial en las entrañas de la tierra, con los bienes culturales escritos a la Prehistoria en las numerosísimas cuevas malagueñas. El primer bloque, y el destinado a transitar entre tumbas de gigante como metafórico modo de presentación del fenómeno del Megatilitismo y el final de la Prehistoria en la provincia malagueña, el segundo [Fig.2]. El especialmente convertido como recurso museográfico por la Tumba del Guerrero, se destinaba a la presentación del período entre Maniate y Malaka, la búsqueda de griegos y fenicios en el litoral malagueño, bloque muy extenso por su especial presencia y su singularidad en nuestra provincia.» (Palomares, 2018, p.382).

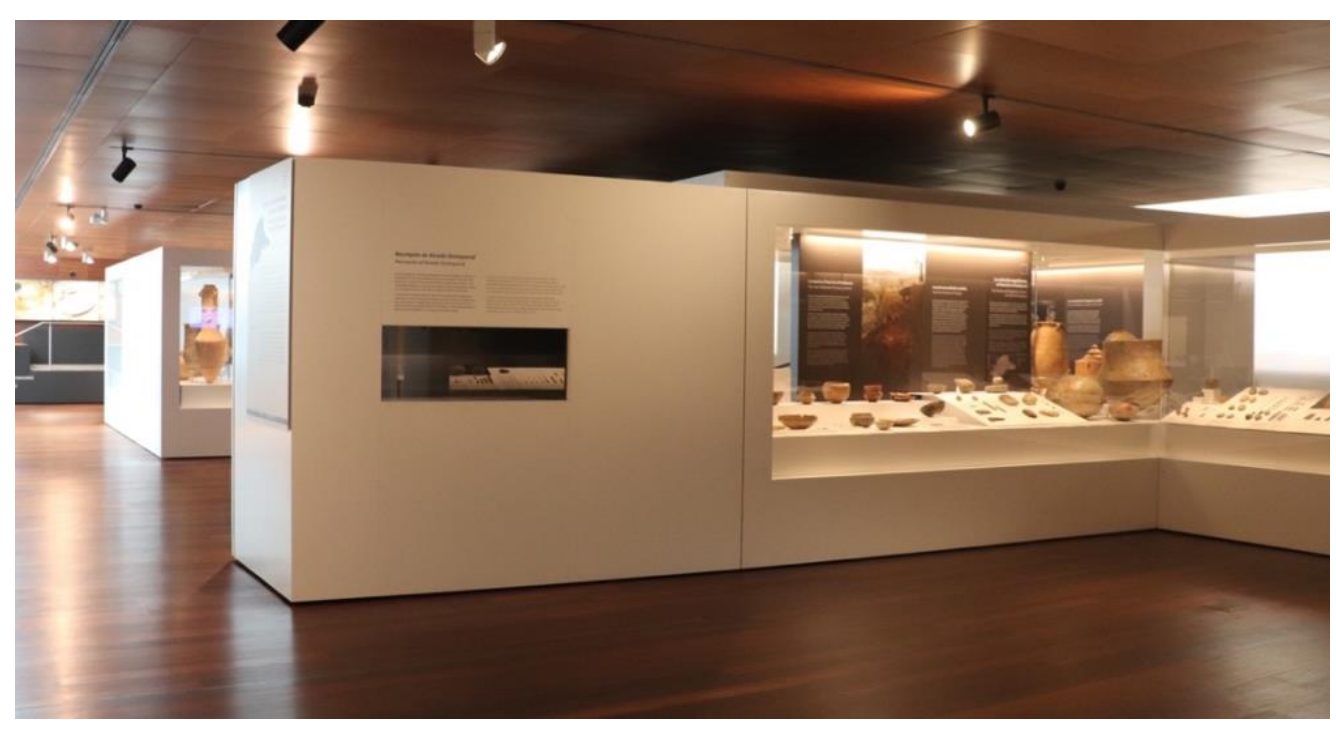

Fig. 2. Vista de diversas salas de la sección de arqueología del Museo de Málaga, elaboración propia, 2020.

A estos, seguían, y siguen, un bloque destinado a la romanización de la provincia y la tardoantigüedad. El recorrido se culmina con un espacio centrado en la arqueología medieval. De este modo podemos destacar que se trata de un lugar del todo reducido tanto para la potencia arqueológica de la provincia como para las características de la museología del s. XXI. 
Esta configuración quedó, acertadamente fracturada, según nuestro punto de vista, con la inserción de la Tumba del Guerrero que, aunque no de manera completa, muestra la unión expositiva de contenedor y contenido necesario para la compresión de la sociedad de su pasado. El encargado de dirigir el proceso de traslado y musealización de los restos fue José Ángel Palomo Semper, Jefe del Departamento de Conservación e Investigación del Museo de Málaga. Así, se le concedió un espacio específico que se adaptase a la comprensión de la tumba tras la intensa labor de restauración de la estructura y el ajuar (Palomares, 2018). Por tanto, podemos señalar que pertenece a la tipología denominada «Traslado a museo» dentro de las presentes en la ciudad de Málaga donde también se da la cubrición, el almacenamiento «in memorian», y la conservación in situ (García González \& Íñiguez, 2019, p.4-5 [Fig. 3].

\begin{tabular}{|c|c|c|c|}
\hline Traslado a museo & Cubrición & $\begin{array}{c}\text { Almacenamiento } \\
\text { “in memoriam" }\end{array}$ & Conservación in situ \\
\hline $\begin{array}{c}\text { Tumba del Guerrero } \\
\text { (Málaga) }\end{array}$ & $\begin{array}{c}\text { Necrópolis de incineración } \\
\text { en Calle Beatas (Málaga) }\end{array}$ & $\begin{array}{c}\text { Necrópolis emiral de } \\
\text { Málaga }\end{array}$ & $\begin{array}{c}\text { Hipogeo fenicio en actual } \\
\text { aparcamiento Alcazaba } \\
\text { (Málaga) }\end{array}$ \\
\hline $\begin{array}{c}\text { Hipogeo de la Calle } \\
\text { Mármoles (Málaga) }\end{array}$ & $\begin{array}{c}\text { Necrópolis de Gibralfaro } \\
\text { (Málaga) }\end{array}$ & $\begin{array}{c}\text { Necrópolis islámicas } \\
\text { del abal de Attabanin } \\
\text { (Málaga) }\end{array}$ & $\begin{array}{c}\text { Mezquitas funerarias y } \\
\text { panteón en la calle Agua } \\
\text { (Málaga) }\end{array}$ \\
\hline
\end{tabular}

Fig. 01: Tabla en la que se enmarca la clasificación general en lo que respecta a las posibles intervenciones tras la excavación de necrópolis o tumbas con la inserción de ejemplos (elaboración propia).

Fig. 3. Tabla que muestra la clasificación general de tipologías de actuación tras el hallazgo de necrópolis o tumbas en la que se presenta la adecuación de la Tumba del Guerrero como ejemplo, García González e Íñiguez, 2019.

Hasta 2014 se planteaba un discurso que aunara las principales tumbas de época protohistórica presentes en el Museo. Nos referimos al hipogeo de la calle Mármoles y la tumba del Guerrero pero, por las características del contenedor, es decir, un edificio histórico del s. XVIII, junto al hecho de situar la sección de arqueología en la segunda planta, no fue factible debido al peso de ambos elementos. Ello lleva a desplazar la inserción de la tumba de la calle mármoles, que hasta 2014 tenía un espacio dentro de la exposición arqueológica, al almacén visitable y que, desgraciadamente lo relega a un último plano «pues como es espacio de tutela de colecciones en almacén no dispone de los necesarios medios auxiliares de contextualización del bien cultural, de los que carece en su actual presentación» (Palomares, 2018, p.383).

Esta situación también impidió que la estructura de la Tumba del Guerrero fuese trasladada al completo y supuso la eliminación de los sillares horizontales, transportándolos 
al almacén en pro de la disposición de una piedra de carácter artificial que aliviase el peso. Otro elemento que se transformó es la unión de la mampostería y los sillares entre los que se inserta un tablero de aglomerado ignífugo e incluso la unión de los sillares que originariamente, dispuestos también a hueso, presentaban piedras de menor tamaño que iban calzando los sillares y que, de nuevo, con el cambio de ubicación han desaparecido de este lugar.

Definitivamente, en el último tramo del bloque destinado a la protohistoria en la provincia, y al final de una de las alas que configuran la distribución en «L» de las piezas de la sección, se decide insertar la tumba dentro de un cubículo elevado de doble acceso -rampa y escalera de tan solo tres peldaños- que fue posible gracias a la elevación en ese lugar de la cubierta para permitir una doble altura. [Fig. 4]

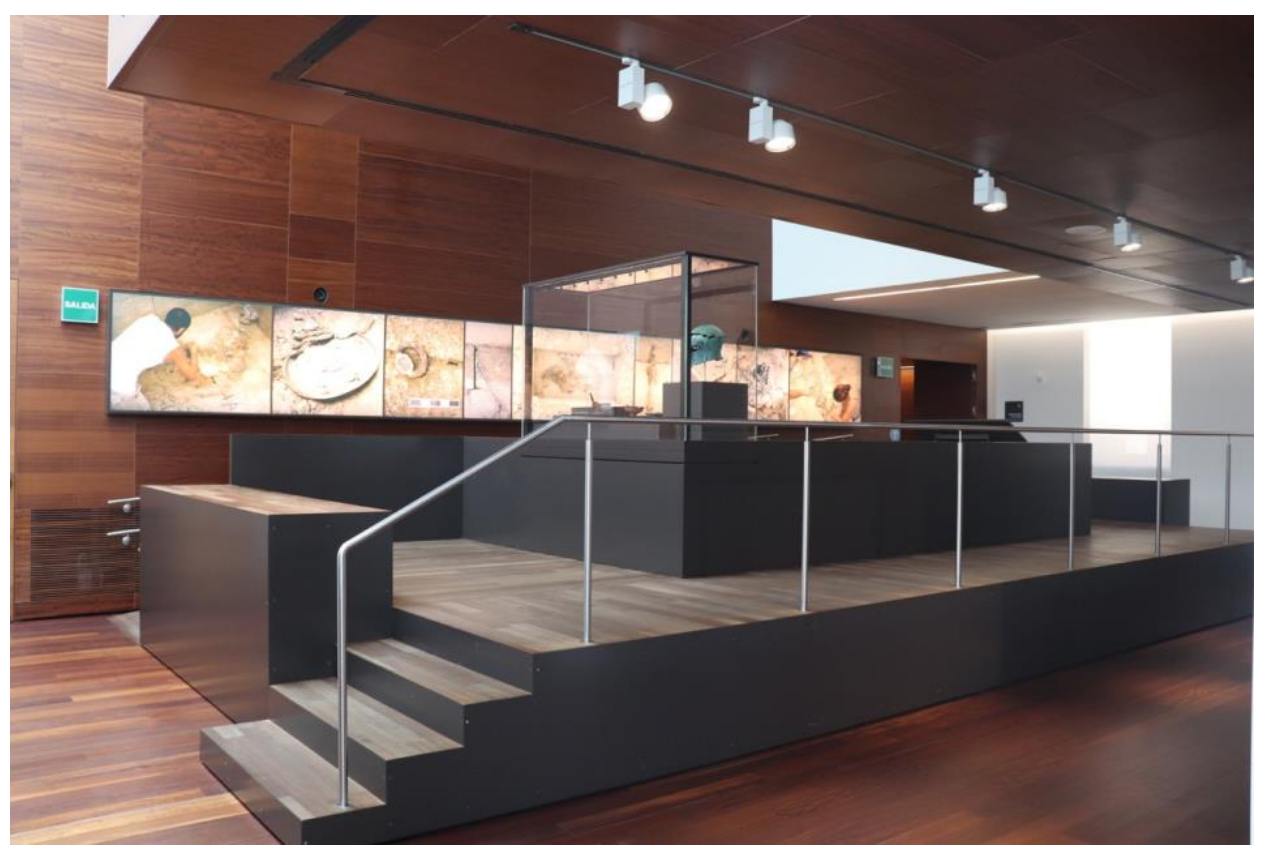

Fig. 4. Vista del espacio destinado a la presentación de la Tumba del Guerrero en el Museo de Málaga, elaboración propia, 2020.

Junto a la estructura, en uno de los lados menores, concretamente el opuesto al pasillo que comunica las salas, se dispone en una vitrina de desarrollo horizontal, parte del ajuar. Específicamente se expone el escarabeo, el quemaperfumes, la phiále de plata, el casco corintio, la punta de lanza de hierro y las varillas de plata, estos últimos elementos situados en un plinto escalonado. [Fig.5] 

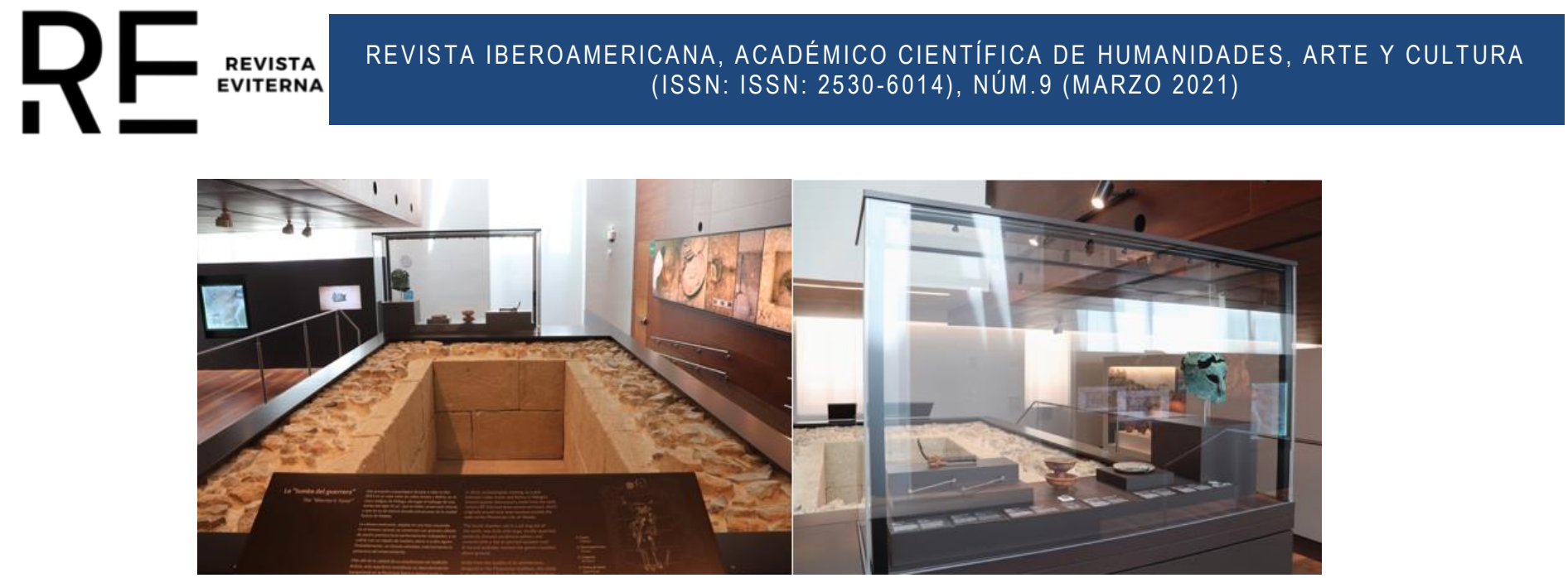

Fig. 5. A la izquierda: Vista de la estructura de la Tumba del Guerrero en el Museo de Málaga tras las modificaciones que supuso sobre la original su traslado y disposición en la segunda planta del Museo, elaboración propia, 2020. A la derecha: Detalle de la vitrina en la que se dispone el ajuar hallado en Tumba del Guerrero en el Museo de Málaga, elaboración propia, 2020.

Por último, la difusión del hallazgo, así como la presentación de elementos no expuestos, se realiza mediante la inserción de fotografías en un panel retroiluminado que discurre horizontalmente en paralelo a la tumba y la disposición de la información en el atril bajo este. A ello habría que añadir la disposición de una pantalla, que hace tiempo dejó de funcionar y que esperamos se encuentre activa en un futuro, en la que se explica la trayectoria del bien desde su hallazgo.

Por tanto, en la disposición actual podemos destacar el hecho de mostrar el continente, es decir, la estructura en cista de la tumba, con parte de su contenido. También sobresale la posibilidad del visitante de interaccionar con el bien mediante la manipulación del casco corintio presente en la unidad tiflológica y la muestra de las fases y detalles de la excavación arqueológica que complementan a la estructura y parte de la cultura material a través del panel retroiluminado. [Fig. 6]

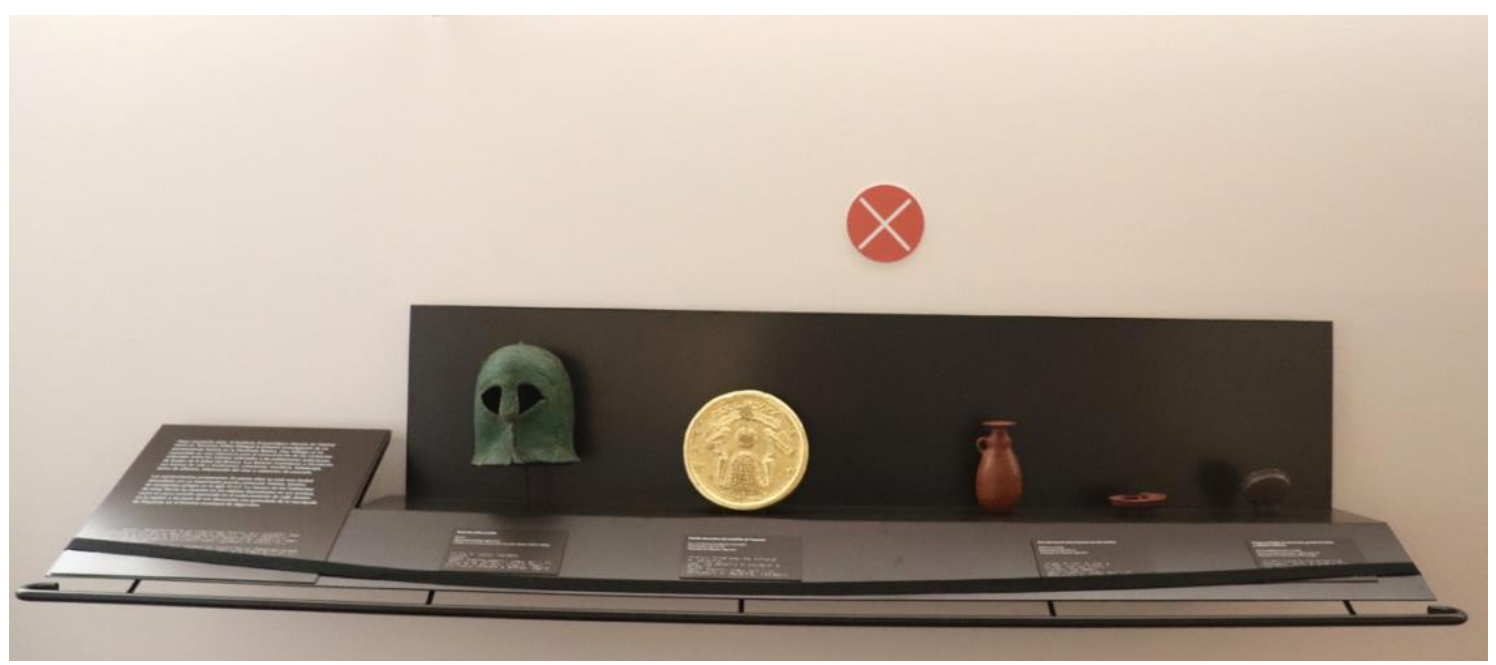

Fig. 6. Vista de la unidad tiflológica en la que se recrea el casco corintio de la Tumba del Guerrero en el Museo de Málaga, elaboración propia, 2020. 


\section{Paralelos}

Si atendemos a la musealización de necrópolis y tumbas cabe precisar que tal y como se señala en recientes publicaciones:

«... han seguido un camino muy diverso con respecto a la musealización de otros espacios como pueden ser hábitat, edificios monumentales, fortificaciones, edificios de ocio, villas en caso del mundo romano, e incluso calzadas o sobre todo elementos defensivos. Dicha musealización empezó a extenderse en la década de los 80 tal y como muestran en sus investigaciones de Sposito (2007, p. 11) Ruggieri Tricoli (2007, p. 7-10) o Ranellucci (2009) y han seguido López-Menchero (2012) Zelli (Medeiro), o García González (García González, 2017)). Ahora bien, hay excepciones en cronologías tempranas como la musealización in situ de las mezquitas funerarias y panteón de calle Agua, en Málaga en los inicios de la década de los 90 o de la necrópolis inserta en el actual Museo Arqueológico Provincial de Cartagena protegido gracias a la labor realizada por Pedro San Martín Moro. Ahora bien, no siempre se procede de este modo y muchas veces las necrópolis se cubren o en la mayoría de las veces se excavan siendo debidamente empaquetados sus elementos (en los mejores casos una vez excavadas al completo, sin que la ciudadanía sea consciente del pasado que pudo tener una calle, una plaza o el subsuelo de una autovía en algún punto remoto) o las estructuras más importantes de la necrópolis o la única tumba hallada en un espacio se traslada a un museo.» (García González \& Íñiguez, 2019, p.3)

Partimos por tanto de la premisa de que la musealización de tumbas y necrópolis es diversa, múltiple y disímil en cuanto a cronología y características. No podemos estudiar del mismo modo estructuras dolménicas y enterramientos, por ejemplo, medievales, en fosas excavadas en la propia tierra. Siendo conscientes de ello, en este apartado mostraremos diversos ejemplos de musealización de tumbas que se mueven en la vertiente objeto de estudio de las establecidas en la fig. 3, pues han sido trasladadas al museo. De los múltiples casos existentes pasamos a presentar tres de ellos. Los dos primeros, el del Museo de las tumbas reales de Sipán en Perú y el Museo de las Antiguas Tumbas Luoyang, por ser la guía en la actualidad de la tutela, estudio, conservación y difusión de necrópolis, y el tercero, por mostrar un ejemplo nacional de traslado de una tumba, concretamente de la tumba de Acilia Plecusa, al Museo de la Ciudad de Antequera.

Como señalábamos, una de las actuaciones mas destacadas de traslado de los materiales constituyentes de una tumba lo encontramos en el complejo que conforman las tumbas reales de Sipán en Perú, pertenecientes a la cultura moche. En el Museo, abierto al público en 2002, la disposición de las estructuras funerarias junto a su ajuar, siguiendo el 
sistema expositivo alemán, se conjuga con la tradición anglosajona al recrear la tumba en las condiciones en las que fue encontrada por los arqueólogos tras el descubrimiento realizado por los expoliadores y la detención policial de estos.

El Museo de las Antiguas Tumbas Luoyang, situado en este municipio dentro de la provincia de Henan, concretamente en el lugar donde se excavaron muchas de las tumbas expuestas, muestra una necrópolis que presenta mas de veinte tumbas pertenecientes a las dinastías Han y Song del Norte, y que por tanto nos permiten acercarnos al mundo de la muerte desde la Edad del Hierro hasta el siglo XIII d.C. Este nos permite visitar veinticinco tumbas de varias dinastías que han sido rehabilitadas y adecuadas para su contemplación. Se presentan por tanto las estructuras con sus correspondientes ajuares.

En cuando al caso, mas cercano, el del Museo de la ciudad de Antequera, debemos señalar que este se encuentra en el Palacio de Nájera desde 1966 pero su origen nos hace retrotraernos a 1908 gracias al trabajo realizado por Don Rodrigo Amador de los Ríos. Fue renovado en 2010 y es en ese momento cuando se decide mostrar la tumba de Acilia Plecusa dentro del área temática «Ritos funerarios de época romana. Vida cotidiana y repertorio escultórico de las villas romanas». El descubrimiento de esta tumba se produce en 1993 en la conocida como necrópolis del paraje conocido como «las maravillas». Se trata de un columbario realizado en sillares de piedra unidos con grapas de plomo en cuyo espacio central se sitúa el sarcófago donde fue enterrada Acilia Plecusa a finales del s. II. d. C, siendo posiblemente su cuerpo el último en disponerse en este espacio familiar, ordenando, tras su fallecimiento que la tumba se sellase. Su situación, prácticamente bajo las vías del tren, hizo inviable su musealización in situ y el columbario fue trasladado a las inmediaciones del que iba a ser Museo de la Prehistoria Andaluza hasta que en 2009 se propone que se inserte dentro del programa museológico del Museo de la ciudad de Antequera. Para su disposición en el museo, al igual que veremos ocurre con la Tumba del Guerrero se desmontan los sillares y se procede su montaje en las instalaciones del museo. Ahora bien, en este caso se dispone el columbario a una cota inferior para que los visitantes lo observen asomándose al espacio abierto prácticamente al nivel de la cubierta. Para su mejor comprensión se dispone un panel que recorre toda la pared y en el que se inserta una ilustración que recrea la tumba.

\section{Propuesta de mejora en el discurso expositivo de la Tumba del Guerrero}

Los discursos museísticos y la museografía que los sustenta no son inmutables, sino que se deben actualizar al menos en dos vertientes interconectadas: en primer lugar, a partir de los 
cambios sociales y, en segundo lugar, a partir de la actualización de las investigaciones, pues ambas cuestiones modifican tanto la demanda de información como la fórmula de difusión. Por tanto, lo que en este apartado venimos a precisar es una propuesta de mejora motivada por estos cambios y no por la propuesta expositiva vigente que fue producto de sus circunstancias y que permitió salvar y difundir uno de los hallazgos más destacados realizados en Málaga. A pesar de que se realizó una importante labor, la publicación del estudio completo y el tiempo que ha pasado desde la propuesta, invita a una reordenación museográfica que permita devolver y acercar los nuevos datos a la sociedad a pesar de ser conscientes de que quizá, todo ello sea, al menos por el momento, debido a cuestiones eminentemente económicas, museografía imposible. [Fig. 7]



Fig. 7. A la izquierda: conjunto de ajuar de la tumba del guerrero tras el proceso de restauración de las piezas A, B y C. A la derecha: arriba, vista cenital de la tumba durante el proceso de excavación y abajo, dibujo arqueológico con la disposición in situ de las piezas que componen el ajuar. Elaboración propia, 2020 a partir de la Fig.6 en García González et alii, 2012 y Navarro Valls, en línea. 
Una de las primeras circunstancias adversas a las que nos enfrentamos es la motivada desde el inicio por la no conservación in situ de la tumba en parte de los solares excavados junto al entramado urbano almohade y la fosa de época moderna. Dicho esto, y una vez procedido al cambio de ubicación y su afortunada disposición en el museo, planteamos una serie de mejoras sustentadas en el desarrollo de las investigaciones y en nuevas experiencias entre el visitante y los bienes en pro de conocer su historia.

Así, consideramos que son posibles mejoras derivadas de la inserción de herramientas basadas en las Tecnologías de la Información y la comunicación ${ }^{1}$ TICs y recursos bibliográficos; modificaciones en el espacio expositivo; inclusión de nuevos materiales; modificación de contenidos de conexión con el público mediante la creación de un programa de actividades específico; la incorporación del mensaje «prohibido no tocan» y la conexión de un circuito que conecte las secciones mediante un hilo conductor: el mundo de la muerte en Málaga.

En cuanto al uso de herramientas que promuevan la comprensión y disfrute de aquello que el visitante está contemplando, reivindicamos la implementación de las TICs en varios sentidos. En primer lugar, sugerimos la disposición de un ordenador en uno de los ángulos de la sala o la presencia de tabletas electrónicas, pues estos instrumentos permitirían mostrar el contenido o la información que todas estas actuaciones arqueológicas, y la investigación fruto de ellas, han generado. Una información de sumo interés que no debe permanecer exclusivamente en reuniones de carácter científico y publicaciones especializadas. Se trata de un patrimonio que pertenece a toda la sociedad, habitantes y visitantes, y debe revertir en ella. Por tanto, la disposición de un ordenador permitiría al visitante interesado ahondar en la información.

Además, este recurso consentirá conectar el bien objeto de estudio con otros hallazgos proptohistóricos de la provincia como el hipogeo del Aparcamiento de la Alcazaba o el de la calle de Campos Elíseos, La rebanadilla en el Cortijo San Isidoro o Cerro del Villar en Málaga; junto a la Necrópolis de Jardín, Los Toscanos, Trayamar, o la Necrópolis de Lagos en Vélez Málaga y el Cortijo de las Sombras en Frigiliana e incluso posibilitará la creación de recorridos alternativos de carácter temático como el que nos hemos atrevido a titular «Qué la tierra te sea leve» en el que mediante la gamificación el visitante podrá ir contemplando espacios relacionados con el mundo de la muerte en Málaga, ya sea mediante

\footnotetext{
${ }^{1}$ En adelante TICs.
} 
la disposición de estructuras o ajuares en la sección arqueológica, en el almacén, o en la sección de Bellas Artes a partir de obras como ;Y tenía corarón! de Enrique Simonet.

De otra parte, no queremos incurrir en el error de centrarnos únicamente en el uso de las TICs, pues no renunciamos que se dispongan herramientas de carácter tradicional como podría ser la inserción de varios libros de la publicación de La Tumba del Guerrero. Un enterramiento excepcional en la Málaga fenicia del siglo VI a.C. Así, si las TICs fallasen durante alguno tiempo y no se dispusiese de medios económicos para repararlas, existiría otro cauce de consulta, también disponible, pese a la duplicidad, para los amantes de los libros en papel, a lo que deberíamos sumar, con objeto de poder desarrollar el conocimiento transversal y temático la realización de una visita guiada bajo el título anteriormente precisado de «Qué la tierra te sea leve».

Para facilitar la comprensión del hallazgo planteamos la proyección de la silueta de los restos óseos y la incorporación paulatina, recreando el ritual de enterramiento, de la silueta del ajuar del mismo modo. Concretamente siguiendo el modelo de uso de proyecciones presente en nuestro ámbito mas cercano. Por ejemplo, en el Museo Arqueológico Provincial de Córdoba, específicamente como recurso para mostrar al visitante los restos arqueológicos del teatro en relación a la estratigrafía y los niveles del mismo, o, fuera de la península, en Assisi, Italia, para presentar el discurrir del agua en las canalizaciones de carácter arqueológico (Cipiciani, 2012).

Y así hemos comenzado a hacer referencia a la cultura material de la tumba. Una vez reflexionado sobre la ubicación de los restos óseos pasamos a plantear modificaciones en cuanto a la relación del público con el ajuar. Al respecto creemos que sería interesante la colación de la recreación del casco corintio para permitir su acceso a invidentes en ese mismo espacio y no a la entrada de la sección. Además, consideramos necesario recrear el casco en escala 1:1 para que los visitantes puedan entender y sentir cual era su peso, tocar el material, observar la presencia del forro que tendría en origen para evitar la reverberación, etc. En definitiva, para conectar al visitante con el bien que está observando y propiciar que, a partir de los sentidos, sientan, como así lo define la Ley 16/1985, de 25 de junio, del Patrimonio Histórico Español que una pieza de esa relevancia constituye su patrimonio. Para mejorar la visión del ajuar apostamos por presentarlo en dos vitrinas diferenciadas. Concretamente en una vitrina de crecimiento vertical, dispondríamos como pieza única el casco corintio y en aquella de desarrollo horizontal mostraríamos a diferente altura, el resto de piezas que configuran el ajuar. 
Por último, proponemos el aprovechamiento de este espacio para demandar una presencia más fuerte de la Tumba del guerrero dentro de las actividades desarrolladas por el museo pues, debido a sus características, propicia que se creen con facilidad sinergias entre los visitantes, la comunidad, diferentes tipos de público y su pasado, la arqueología, y el propio museo. En definitiva, permite vehicular los espacios funerarios de la ciudad y conectar a sociedades de diferentes épocas que, en su gran mayoría habitan o han habitado un mismo lugar.

Ahora bien, como señalábamos en párrafos anteriores, somos conscientes de que, al menos por ahora, se trata de una propuesta museográfica imposible o quizá del primer paso de un camino de renovación. Este deberá ser incluido en la planificación y programación presupuestaria del Museo de Málaga, así como someterse a estudio detallado por parte del equipo del museo ya que supone un gran esfuerzo económico en el que no se contemplan las opciones de una baja inversión pues la inserción de las TICs eleva la cuantía presupuestaria tanto en su creación como en su mantenimiento. Sin embargo, la contrapartida, es decir, el mantener vivo, actualizado y mejorar la comunicación entre la sociedad y su pasado, hace necesaria esta primera aproximación que nos acerca a un individuo «... extranjero en Malaka, pero bien considerado dentro de ella, y tal vez por aquellos sobre los que ejercía una autoridad que [...] han reunido una selección de los objetos que había poseído en vida y marcaban su actividad principal, la guerra, y los han depositado con cuidado e, incluso, devoción» (Domínguez, 2018, 416). Devoción que debe seguir manteniéndose en la actualidad hacia este importante ejemplo de procesos de movilidad social y confluencia de culturas.

\section{Referencias bibliográficas}

Cipiciani, Ma Letizia (2012). Assisi, la città che non c'e. MLC: Perugia.

Chicharro Chamorro, José Luis (2013), Los museos y la Diputación de Jaén. Boletín. Instituto de Estudios Giennenses, nº 207, pp. 1167-1184.

De la Sierra, Juan Alonso (2017), Museo Arqueológico Provincial de Cádiz (1887-1970). Boletín del Museo Arqueológico Nacional, n³5, pp. 29-42.

Domínguez Monedero, Adolfo J. (2018), El ritual funerário de la Tumba del Guerrero de Málaga en el contexto del siglo VI a.C., En: David García González, Sonia López Chamizo 
y Eduardo García Alfonso (ed.). La Tumba del Guerrero. Un enterramiento excepcional en la Málaga fenicia del s. VI a.C. (pp. 395 - 420). Consejería de Cultura de la Junta de Andalucía.

García González, Julia (2016). Arquitectura contemporánea y arqueología: intervenciones en el patrimonio (1985-2010). (Tesis de Doctorado). Recuperado de: http://digibug.ugr.es/handle/10481/43879

García González, Julia (2017). Yacimientos arqueológicos y arquitectura contemporánea. Intervenciones en el Patrimonio. Universidad de Granada: Granada.

García González, David; Adroher Auroux, Andrés Mª y García González, Julia (2018), El quemaperfumes de la Tumba del Guerrero de Málaga, En: David García González, Sonia López Chamizo y Eduardo García Alfonso (ed.). La Tumba del Guerrero. Un enterramiento excepcional en la Málaga fenicia del s. VI a.C. (pp. 269 - 276). Consejería de Cultura de la Junta de Andalucía.

García González, Julia; Dorado Alejos, Alberto y Adroher Auroux, Andrés Ma (2018), Materiales cerámicos procedentes del nivel de colmatación de la Tumba del Guerrero de Málaga, En: David García González, Sonia López Chamizo y Eduardo García Alfonso (ed.). La Tumba del Guerrero. Un enterramiento excepcional en la Málaga fenicia del s. VI a.C. (pp. 277 287). Consejería de Cultura de la Junta de Andalucía.

García González, David; López Chamizo, Sonia; Cumpián Rodríguez, Alberto; y Sánchez Bandera, Pedro Jesús (2013), Mainake, nº XXXIV, pp. 277-292.

García González, David y García Alfonso, Eduardo (2018), La phiále de la Tumba del Guerrero de Málaga, En: David García González, Sonia López Chamizo y Eduardo García Alfonso (ed.). La Tumba del Guerrero. Un enterramiento excepcional en la Málaga fenicia del s. VI a.C. (pp. 239 - 250). Consejería de Cultura de la Junta de Andalucía.

García González, David; López Chamizo, Sonia; Cumpián Rodríguez, Alberto; y Moreno Ortega, Ana Belén (2018), El lugar del hallazgo de la Tumba del Guerrero de Málaga. Caracterización arqueológica del solar de calle Jinetes, En: David García González, Sonia López Chamizo y Eduardo García Alfonso (ed.). La Tumba del Guerrero. Un enterramiento excepcional en la Málaga fenicia del s. VI a.C. (pp. 75 - 107). Consejería de Cultura de la Junta de Andalucía.

García González, Julia e Íñiguez, Carmen (2019), De la excavación a la exposición temporal como recurso educativo y turístico. Propuestas sobre la divulgación de la cultura funeraria 
desde la protohistoria a la época islámica en Málaga», En: XX Encuentro de cementerios patrimoniales: Los cementerios como recurso cultural, turístico y educativo, (pp. 1-21).

Guisante Santamaría, Pablo (2017), Hacia el centenario del Museo de Huelva. Boletín del Museo Arqueológico Nacional, no 35, pp. 309-321.

Gutiérrez de Pablo, Carlos (2013). El Palacio de la Aduana de Málaga como imagen de la historia de la ciudad. (Tesis de doctorado). Universidad de Málaga, Málaga, España.

Lamarca Irisarri, Daniel; Rozalén, Marisa y Lozano, José Antonio (2018), El marfil de la Tumba del Guerrerode Málaga: caracterización geoquímica, En: David García González, Sonia López Chamizo y Eduardo García Alfonso (ed.). La Tumba del Guerrero. Un enterramiento excepcional en la Málaga fenicia del s. VI a.C. (pp. 261 - 268). Consejería de Cultura de la Junta de Andalucía.

Jiménez-Brobeil, Sylvia y Laffranchi, Zita (2018), El estudio antropológico de la Tumba del Guerrero de Málaga, En: David García González, Sonia López Chamizo y Eduardo García Alfonso (ed.). La Tumba del Guerrero. Un enterramiento excepcional en la Málaga fenicia del s. VI a.C. (pp. 301 - 310). Consejería de Cultura de la Junta de Andalucía.

López Chamizo, Sonia (2018), Estudio bioarqueológico de la tumba del Guerrero de Málaga. Apuntes tafonómicos, En: David García González, Sonia López Chamizo y Eduardo García Alfonso (ed.). La Tumba del Guerrero. Un enterramiento excepcional en la Málaga fenicia del s. VI a.C. (pp. 301 - 310). Consejería de Cultura de la Junta de Andalucía.

López-Menchero Bendicho, Víctor Manuel (2012). Manual para la puesta en valor del patrimonio arqueológico al aire libre. Trea: Gijón.

Navarro Ortega, Ana; San Martín Montilla, Concepción; y Camacho Moreno, Manuel (2017), Museo Arqueológico de Sevilla (1879-2017): 138 años de historia y algunos mas, Boletín del Museo Arqueológico Nacional, $\mathrm{n}^{\circ}$ 35, pp. 439-458.

Navarro Valls, Juan. V. (s.f), La ciudad fenicia y púnica de Málaga. Recuperado de http://historiartemalaga.com/malaka.html

Núñez Pariente de León (2018), Las varillas de plata de la Tumba del Guerrero de Málaga, En: David García González, Sonia López Chamizo y Eduardo García Alfonso (ed.). La Tumba del Guerrero. Un enterramiento excepcional en la Málaga fenicia del s. VI a.C. (pp. 231 - 238). Consejería de Cultura de la Junta de Andalucía. 
Ortiz García, Jónatan y Jiménez Higueras, Ángeles (2018), El anillo con escarabeo de la Tumba del Guerrero de Málaga, En: David García González, Sonia López Chamizo y Eduardo García Alfonso (ed.). La Tumba del Guerrero. Un enterramiento excepcional en la Málaga fenicia del s. VI a.C. (pp. 251 - 260). Consejería de Cultura de la Junta de Andalucía.

Palomares, José Ángel (2018), La musealización de la Tumba del Guerrero, una aproximación a su singularidad expositiva o cómo se hace un museo, En: David García González, Sonia López Chamizo y Eduardo García Alfonso (ed.). La Tumba del Guerrero. Un enterramiento excepcional en la Málaga fenicia del s. VI a.C. (pp. 379 - 391). Consejería de Cultura de la Junta de Andalucía.

Quesada, Fernando y García González, David (2018), Las armas de la Tumba del Guerrero de Málaga, En: David García González, Sonia López Chamizo y Eduardo García Alfonso (ed.). La Tumba del Guerrero. Un enterramiento excepcional en la Málaga fenicia del s. VI a.C. (pp. 145 - 230). Consejería de Cultura de la Junta de Andalucía.

Ramos Lizana, Manuel (2017), El Museo de Almería. ¿Otra historia o la misma historia de siempre?. Boletín del Museo Arqueológico Nacional, nº 35, pp. 5-23.

Ranellucci, Sandro (2009). Coperture archeologiche. Allestimenti protettivi sui siti archeologici. Dei: Roma.

Ruggieri Tricoli, MC (2007), Musei sulle rovine: presentazione di una ricerca parziale», En M.C., Ruggieri Tricoli (ed.). Musei sulle rovine Architetture nel contesto archeologico, (pp. 7-10), Edizioni Lybra immagine, Milán.

Santana Guzmán, Antonio Jesús (2019), Turismo, Cultura y Patrimonio: Intervenciones en Málaga, ciudad de museos, En J.L. Lerma García; V.M. López Menchero Bendicho y A. Maldonado Zamora (ed.) I Simposio anual de Patrimonio Natural y Cultural ICOMOS España 21 23 de noviembre 2019 (Madrid), (pp. 543-550), Universitat Politècnica de València, Valencia.

Sposito, Alberto (2007), Il paesaggio come prospettiva della musealizzazione archeologica europea, En: M.C. Tricoli (ed.). Musei sulle rovine Architetture nel contesto archeologico, (pp. 6-11). Edizioni Lybra Immagine, Milán.

Toro Moyano, Isidro (2017), El Museo Arqueológico de Granada. Pasado, presente y futuro. Boletín del Museo Arqueológico Nacional, n³5, pp. 253-265.

Zelli, Flavia (2013). Oltre la rovina. Il progetto contemporaneo in ambito archeologico. (Tesis de doctorado). Universidad de Valladolid, Valladolid, España. 